\title{
A Note on a Paper of S.G. Kim
}

\author{
Diana M. Serrano-Rodríguez \\ Departamento de Matemáticas, Universidad Nacional de Colombia \\ 111321 - Bogotá, Colombia \\ dmserrano0@gmail.com, diserranor@unal.edu.co
}

Abstract: We answer a question posed by S.G. Kim in [3] and show that some of the results of his paper are immediate consequences of known results.

Key words: Bohnenblust-Hille inequality.

AMS Subject Class. (2000): 46G25, 30B50.

The recent paper [3] deals with extreme multilinear forms and polynomials and the constants of the Bohnenblust-Hille inequalities. In this note we answer a question posed in [3] and show that two theorems stated in [3] are immediate corollaries of well known results of this field.

Let $\mathbb{K}$ be $\mathbb{R}$ or $\mathbb{C}$. The multilinear Bohnenblust-Hille inequality asserts that, given a positive integer $m$, there is an optimal constant $C(m: \mathbb{K}) \geq 1$ such that

$$
\left(\sum_{i_{1}, \ldots, i_{m}=1}^{\infty}\left|U\left(e_{i_{1}}, \ldots, e_{i_{m}}\right)\right|^{\frac{2 m}{m+1}}\right)^{\frac{m+1}{2 m}} \leq C(m: \mathbb{K})\|U\|,
$$

for all bounded $m$-linear forms $U: c_{0} \times \cdots \times c_{0} \rightarrow \mathbb{K}$. The case of complex scalars was first investigated in [1] and the case of real scalars seems to have been just explored more recently. It is well known that the exponent $2 m /(m+1)$ is sharp, so one of the main goals of the research in this field is to investigate the constants involved. The following result was proved in [4]:

Theorem 1. ([4, Corollary 5.4], 2018) Let $m \geq 2$ be a positive integer. If the optimal constant $C(m: \mathbb{R})$ is attained in a certain $T: c_{0} \times \cdots \times c_{0} \rightarrow$ $\mathbb{R}$, then the quantity of non zero monomials of $T$ is bigger than $4^{m-1}-1$.

As an immediate corollary we conclude that if $N_{1}, \ldots, N_{m} \geq 1$ are positive integers such that

$$
\prod_{j=1}^{m} N_{j} \leq 4^{m-1}-1
$$


then

$$
\sup \left(\sum_{i_{1}, \ldots, i_{m}=1}^{N_{1}, \ldots, N_{m}}\left|T\left(e_{i_{1}}, \ldots, e_{i_{m}}\right)\right|^{\frac{2 m}{m+1}}\right)^{\frac{m+1}{2 m}}<C(m: \mathbb{R}),
$$

where the sup runs over all norm one $m$-linear forms $T: \ell_{\infty}^{N_{1}} \times \cdots \times \ell_{\infty}^{N_{m}} \rightarrow \mathbb{R}$. In particular,

$$
\sup \left(\sum_{i, j, k=1}^{2}\left|T\left(e_{i}, e_{j}, e_{k}\right)\right|^{\frac{6}{4}}\right)^{\frac{4}{6}}<C(3: \mathbb{R})
$$

where the sup runs over all norm one $m$-linear forms $T: \ell_{\infty}^{2} \times \ell_{\infty}^{2} \times \ell_{\infty}^{2} \rightarrow \mathbb{R}$, and this is the content of [3, Theorem 4.9].

The polynomial Bohnenblust-Hille inequality for real scalars asserts that, given a positive integer $m$, there is an optimal constant $C_{p}(m: \mathbb{R}) \geq 1$ such that

$$
\left(\sum_{|\alpha|=m}\left|a_{\alpha}\right|^{\frac{2 m}{m+1}}\right)^{\frac{m+1}{2 m}} \leq C_{p}(m: \mathbb{R})\|Q\|,
$$

for all $N \geq 1$ and for all $m$-homogeneous polynomials $Q: \ell_{\infty}^{N}(\mathbb{R}) \rightarrow \mathbb{R}$ given by

$$
Q(z)=\sum_{|\alpha|=m} a_{\alpha} z^{\alpha} .
$$

To the best of our knowledge, the case of real scalars became unexplored until the publication of the paper [2] in 2015, where it is proved that the constants $C_{p}(m: \mathbb{R})$ cannot be chosen with a sub-exponential growth. More precisely,

Theorem 2. ([2, Theorem 2.2], 2015)

$$
C_{p}(m: \mathbb{R})>\left(\frac{2 \sqrt[4]{3}}{\sqrt{5}}\right)^{m}>(1.177)^{m},
$$

for all positive integers $m \geq 2$.

The above result is, obviously, by far, rather precise than [3, Theorem 4.5], which states that

$$
C_{p}(m: \mathbb{R}) \geq 2^{\frac{m+1}{2 m}},
$$


for all positive integers $m \geq 2$. The only case that deserves a little bit more of attention is the case $m=2$, since

$$
\left(\frac{2 \sqrt[4]{3}}{\sqrt{5}}\right)^{2}<2^{\frac{3}{4}}
$$

but in the case $m=2$ a quick look at the proof of [2, Proof of Theorem 2.2] shows that

$$
C_{p}(2: \mathbb{R}) \geq \frac{3^{\frac{3}{4}}}{\frac{5}{4}} \approx 1.8236>2^{\frac{3}{4}},
$$

and this answers in the negative the Question (2) posed by the author in [3, Question (2)].

\section{REFERENCES}

[1] H.F. Bohnenblust, E. Hille, On the absolute convergence of Dirichlet series, Ann. of Math. (2) 32 (1931), 600-622.

[2] J.R. Campos, P. Jiménez-Rodríguez, G.A. Muñoz-Fernández, D. Pellegrino, J.B. Seonne-Sepúlveda, On the real polynomial Bohnenblust-Hille inequality, Linear Algebra Appl. 465 (2015), 391-400.

[3] S.G. KIM, The geometry of $\mathcal{L}\left({ }^{3} \ell_{\infty}^{2}\right)$ and optimal constants in the BohnenblustHille inequality for multilinear forms and polynomials, Extracta Math. 33 (1) (2018), $51-66$.

[4] D. Pellegrino, E. Teixeira, Towards sharp Bohnenblust-Hille constants, Commun. Contemp. Math. 20 (2018), 1750029, 33 pp. 
